\title{
Flame Inclination with Induced Wind through Inlet Opening in a Tall and Narrow Atrium
}

\author{
H. SATOH and H. KURIOKA \\ Kajima Technical Research Institute \\ Kajima Corp. \\ 2-19-1 Tobitakyu, Chofu-shi, Tokyo 182, Japan \\ O. SUGAWA \\ Center for Science and Technology, Science University of Tokyo \\ 2641 Yamasaki, Noda, Chiba 278, Japan
}

\begin{abstract}
Inclination behavior of flames affected by the induced door jet(s) into a tall and narrow atrium space was studied experimentally. The inlet opening(s) were arranged to simulate the configuration of one door and two doors. Induced air velocity through door(s) was estimated considering stack effect and atrium space configuration. In the case where the separation between two doors is smaller than twice width of a door, door jets merged and affected the flame resulting the flame inclination, similar to the single door jet effects. The distribution of the door jet, not at and near the inlet opening but in the atrium space, showed the normal distribution curve. Those of merged door jets demonstrated the additivity of the configurational contribution based on the setting locations of doors. A simple model which gives the estimation of the attacking door jet velocity on a flame is presented considering door configurations, location and size of a fire source, heat release rate, and space factor of an atrium space.
\end{abstract}

Key Words: Atrium, Door Jets, Flame Inclination, Froude Number, Dimensionless heat release rate

\section{Introduction}

In the previous paper [1], we have characterized the atrium space into three groups by space factor $\mathrm{C}$ defined by volume and aspect ratio of $\mathrm{H}^{2} / \mathrm{A}$. One of the groups of atrium space is a tall and narrow configuration. This configuration may have high hazardous potential on fire safety since it may work like a stack when a fire occurs at the bottom. It is this geometry we would like to concern in this paper. We pursued to establish a simple method to evaluate temperature rise in a tall and narrow atrium and the inlet-air velocity at openings during the early stage of a fire for natural and/or forced ventilation condition [1]. We have revealed the relationships among a number of factors; space factor, dimensionless heat release rate, Froude number, and temperature rise in the space. In the process of carry- 
ing out the experiments in a reduced and full scale experiments, we found that the induced door jet(s) played important roles not only on the flame deformation (i.e. extension, expansion, inclination of a flame) but also on the air entrainment into flame. The induced door jet also had some effect on the flame resulting in a temperature reduction and limiting height of the plume. The effect of door jet(s) on a flame is a very important factor which correlates to the entrainment behavior and the definition of buoyancy at the source. The effect of wind on the flame inclination has been studied experimentally and theoretically by $\mathrm{Ha}$ mada [2] and Thomas [3] based on Froude Modeling. Later, Escuidier [4],

Brzustowski [5] and Raj [6], and Quintiere [7] presented the inclination behavior based on an effluent model from a stack, and on the extended turbulent diffusion flame. These theoretical model considered entrainment to a plume, and the balance between inertia and buoyancy from a square or a round source. We have discussed the quantitative correlation between induced mass flux into a tall and narrow atrium space and heat release rate [1], and pointed out there were various flame inclination patterns which were produced by induced air. Flame inclination may impact escape potentials. evacuation is through the doors of atrium floor level. The flame inclination behavior which is affected directly or indirectly with door jet(s) is discussed.

Based on the combination of mass, momentum, and energy conservation, we established the correlation between Froude number and heat release rate considering atrium space factor. FIGURE 1 shows this relation obtained from the experiments which were carried out in the full scale atrium of three different buildings and in the reduced scale model. The dimensions and configurations of these three full scale atrium spaces are described in TABLE 1. All plots in FIGURE 1 tend to gather on a line which is parallel and below the theoretical line of adiabatic condition. Effectiveness of the correlation illustrated in FIGURE $1, \mathrm{Fr}_{\mathrm{d}}{ }^{1 / 2}$ against $\mathrm{C}^{1 / 2} \cdot \mathbf{Q}_{\mathrm{fd}}^{* 1 / 3}$, was determined using data from three different full 
TABLE 1 Dimension and ventilation methods of the atrium spaces

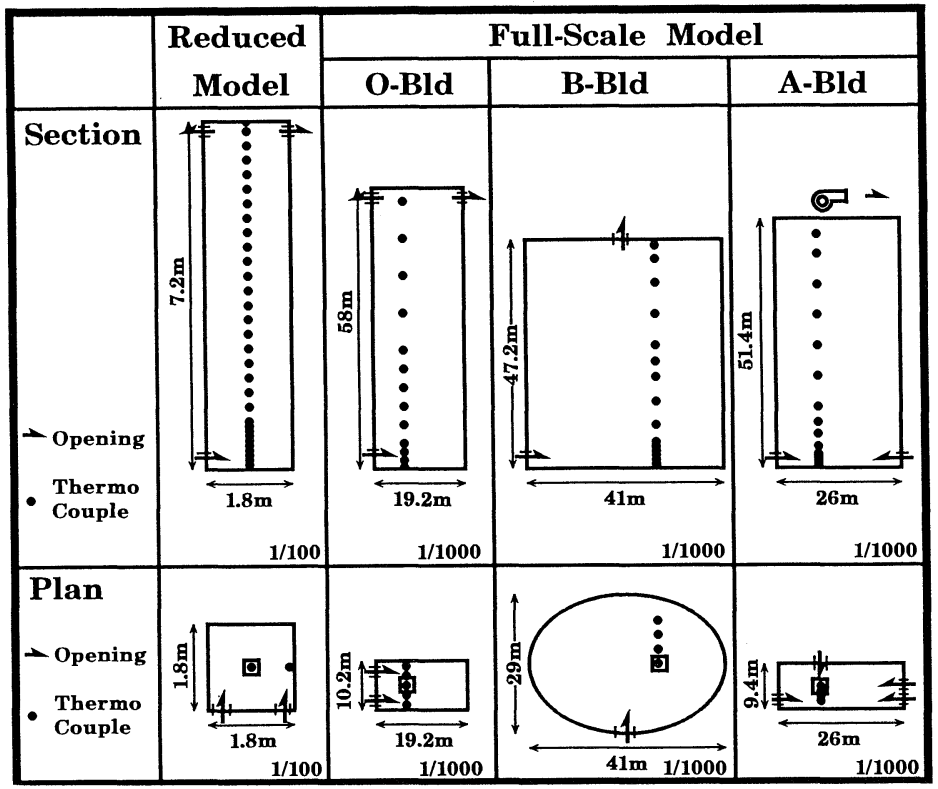

scale atrium spaces and the reduced scale model. In summary, this correlation is used to estimate the door jets velocity under the designed fire size (heat release rate) and given configuration of atrium space.

2. Flame Inclination in Open In order to understand the inclination behavior of a flame attacked by external wind in an open space, a $10 \mathrm{~cm}$ $x 10 \mathrm{~cm}$ square gas diffusion burner was located downstream for both with and without a floor. Flame inclination angles were estimated changes in wind velocity and heat release rate. FIGURE 2 shows representative results of the

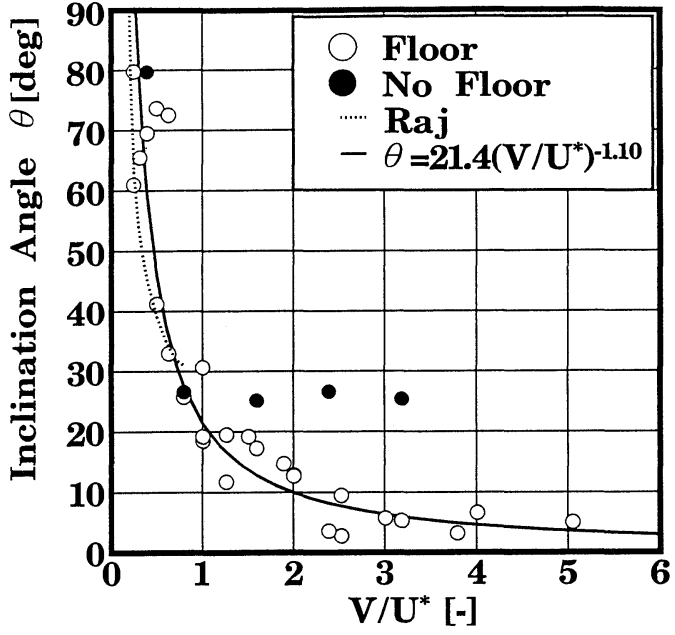

FIGURE 2 Relation between inclination angle of a diffusion flame and horizontal wind velocity in dimensionless expression. Wind velocity is normalized by heat release rate based velocity. 
inclination angle as a function of dimensionless wind velocity, $\mathrm{V} / \mathrm{U}^{*}$, where $\mathrm{V}$ is horizontal wind velocity and $\mathrm{U}^{*}$ is the dimensional upward velocity based on heat release rate (defined as $\left.\mathrm{U}^{*}=\left(\mathrm{gQ} / \mathrm{b}_{\mathrm{o}} \rho_{\infty} \mathrm{T}_{\infty} \mathrm{Cp}_{\infty}\right)^{1 / 3}\right)$. The dimensionless wind velocity is a variable for correlating the measured flame inclination angle. A similar variable was used by Thomas [3] and Quintiere [7]. The flame inclination angle was evaluated by the height where the highest temperature appeared in the array of thermocouples located $20 \mathrm{~cm}$ apart in the downwind stream. For the light wind case, it is difficult to determine the highest temperature position, which results in the uncertainties on the evaluation of flame angle. In the case of no floor, the flame angle terminated around 30 degrees. For the case with floor, the flame inclination approached to almost ground level. Based on the data, the flame inclination angle with a floor is expressed empirically as;

$$
\theta=21.4\left(\mathrm{~V} / \mathrm{U}^{*}\right)^{-1.1} \text {. }
$$

A similar empirical curve was also given by Raj [6] and is illustrated in FIGURE 2. Within the range where dimensionless wind velocity is smaller than 0.8 , the flame inclination appeared to terminate at about 30 degrees as shown in FIGURE 8 of reference [7]. However, beyond this range, the flame inclination angle seemed to approach ground level, and the empiri-

cal equation (1) was validated.

3. Flame Inclination in an Atrium

3.1 Visualization of Door Jet(s) and Concept of Flow Dynamic

Experiments were carried out using the reduced scale model of atrium space which was used for the previous tests [1] having $1.8 \mathrm{~m} \mathrm{x}$ $1.8 \mathrm{~m}$ (of floor area) $\mathrm{x}$ $7.2 \mathrm{~m}(\mathrm{H})$. Several configuration designs for inlet opening(s) were adopted for the tests. However, in this paper, we dealt with the cases of one

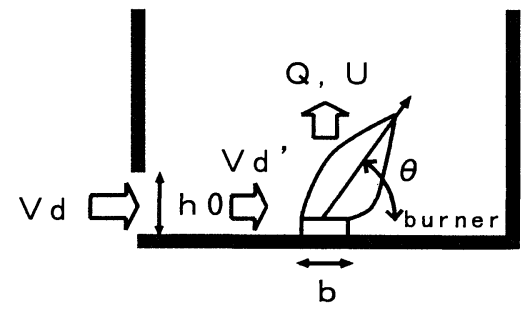

FIGURE 3 (a) Conceptual flame inclination affected by the induced door jet in vertical cross section.

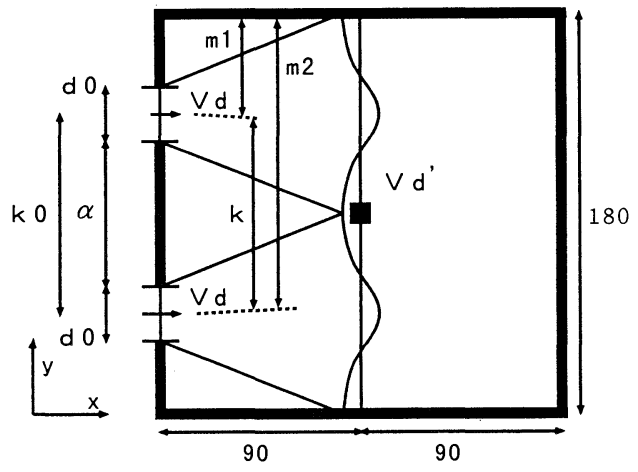

FIGURE 3 (b) Flow patterns of two induced jet and merging and attacking on the flame. Definition of door jet flow, $V_{d}$, position to express the width of the jet flow, door width, $d_{o}$, and door separation distance, $\alpha$, are illustrated. 
door centered and of two doors having the half width of the one door and which were symmetrically distributed on a same side of a model as shown FIGURE 3(b). Except for an initial transient duration, the thermal and flow characteristics of the door jet(s) showed some fluctuations but were reasonably steady. A typical response of flame inclination by induced door jet(s) and also flow pattern of door jets are presented in FIGURE 3(a) and 3(b), respectively. The inclined flame angle, $\theta$, adopted as $\theta=90^{\circ}$ for vertical flame as the reference, was estimated based on eye-observations and recorded images of photo and video systems. The velocity distributions of the door jet(s) were obtained at $5 \mathrm{~cm}, 45 \mathrm{~cm}$, and $80 \mathrm{~cm}$ (or $110 \mathrm{~cm}$ ) downstream from the opening(s) based on the visualized flow by a smoke-wire system. Liquefied paraffin was coated on a electric wire (a Ni-Cr alloy) to produce fine vaporized particles when the electric wire was charged. This system was employed as the smoke-wire method to visualize the door jets. Distribution of the door jet showed almost a normal distribution, as illustrated in FIGURE 3(b), except at and near the door downstream. The visualized profile of the door jet through one opening was evaluated and translated into numeric data at every $1 \mathrm{~cm}$ space. The digitized profile was compared with the normal distribution curve, having characteristic width, $\sigma$, and induced jet velocity, $\mathrm{V}_{\mathrm{d}}$, corresponding to the measured points along the smoke-wire. The distribution profile was then expressed empirically as;

$$
\mathbf{v}_{\mathrm{d}}^{\prime}=\frac{\mathbf{k} \cdot \mathbf{V}_{\mathrm{d}}}{\sqrt{2 \pi \sigma} \sigma} \exp \left[-\frac{\left(\mathrm{y}-\mathbf{m}_{\mathbf{i}}\right)^{2}}{2 \sigma^{2}}\right] \text {, }
$$

where $\mathrm{k}$ is experimentally determined constant, and $\mathrm{y}$ and $\mathrm{m}_{\mathrm{i}}$ are defined in FIGURE 3 (b) as the traveling distance for downward and mean value of the door jet velocity, respectively. When two doors were set, two normal distributions of door jets were observed and each profile was able to be expressed by equation (2). Two jets merged with each other when the separation distance between two doors was smaller than the two times wide of a door. When the separation distance between two doors was greater than twice wide of one door opening, little merging was observed and they looked like two parallel jets before they passed away from the fire source.

\subsection{Modes of Flame Inclination}

FIGURE 4 shows the typical flame inclination shapes for a steady state $12 \mathrm{~kW}$ fire. The inclination angles of these flames were estimated. In these Figures, flames are blocked by (a), (b), and (c) due to the door configuration. In the block (a), the "center series" of the tests, one door was set at the center of the front wall. The induced door jet collides directly with the flame. The door width was systematically changed from $5 \mathrm{~cm}$ to $180 \mathrm{~cm}$. The block (b) was for the tests called " $\alpha$-series". Two openings, each $1 / 2$ width of one opening set in the block (a), were set with the separation of $\alpha$ between two openings changing from $0 \mathrm{~cm}$ to $120 \mathrm{~cm}$. The block (c), called " $\beta$-series" has one opening which locates off center of the front wall (near a side wall), so that the induced door jet attacks the flame indirectly. In this paper, we consider the cases of (a) and (b) as typical opening configurations which the door jet behavior in case of block (c) will be discussed elsewhere. 


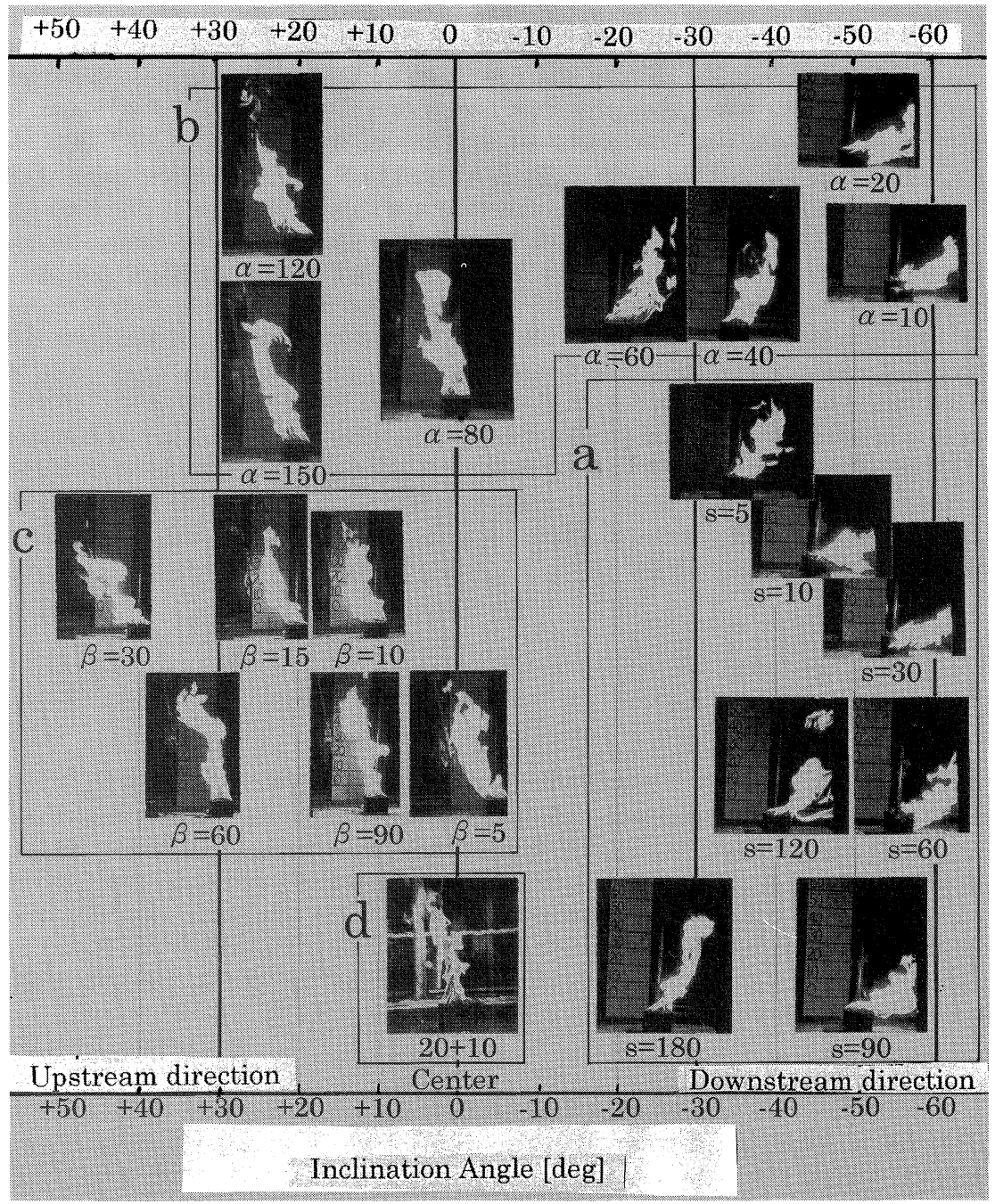

FIGURE 4 Typical flame shapes and inclinations as the door width and separation changed. Block (a) are obtained using a door centered, block (b) two doors and (c) one door located end of the wall. 
4. Decreasing Mode of Door Jets(s) along the traveling distance

\subsection{Model}

In order to estimate the door jet velocity which attacks the flame, we made a dimensional analysis based on the Froude modeling considering the space factor term. First, we assumed the upward velocity, $\mathrm{U}^{*}$, based on the heat release rate following the classical method:

$$
\mathbf{U}^{*}=\left(\frac{\dot{\mathbf{Q}} \cdot \mathbf{g}}{\mathbf{b}_{\mathbf{0}} \cdot \mathbf{C}_{\mathbf{p}} \cdot \boldsymbol{\rho}_{\infty} \cdot \mathbf{T}_{\infty}}\right)^{1 / 3}=2^{1 / 3} \cdot \mathbf{Q}_{\mathbf{b}}^{* 1 / 3} \cdot \sqrt{\mathbf{g} \cdot \mathbf{b}}
$$

It is well known that the jet velocity decrease with increase of $1 / 2$ power of traveling distance in the first phase of the downward stream. Here we assumed the induced door jet velocity, $\mathrm{V}_{\mathrm{d}}$, at traveling distance, $\mathrm{x}$, from the opening can be expressed as;

$$
\mathbf{V}_{\mathbf{d}}=\frac{\mathbf{A}}{\sqrt{\mathbf{x} / 2 \cdot \mathbf{d}_{\mathbf{o}}}} \mathbf{V}_{\mathbf{d}}
$$

where $V_{d}=B \cdot C^{1 / 2} \cdot Q_{f d}^{* / 3} \cdot \sqrt{2 \cdot g \cdot d_{o}}$, and $V_{d}{ }^{\prime}$ can be rewritten using the heat release rate of the source considering inlet opening size and fire size;

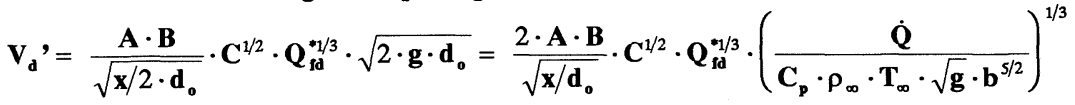

$$
\begin{aligned}
& =\frac{2 \cdot A \cdot \mathbf{B}}{\sqrt{\mathbf{x} / \mathbf{d}_{\mathbf{0}}}} \cdot \mathbf{C}^{1 / 2} \cdot \sqrt{\mathbf{g} \cdot \mathbf{d}_{\mathbf{0}}} \cdot \mathbf{Q}_{\mathbf{b}}^{* / 3} \cdot\left(\frac{\mathbf{b}}{2 \cdot \mathbf{d}_{\mathbf{0}}}\right)^{5 / 6}
\end{aligned}
$$

Considering the space factor of the atrium and heat release rate, the dimensionless door jet velocity which attacks the flame can be expressed as;

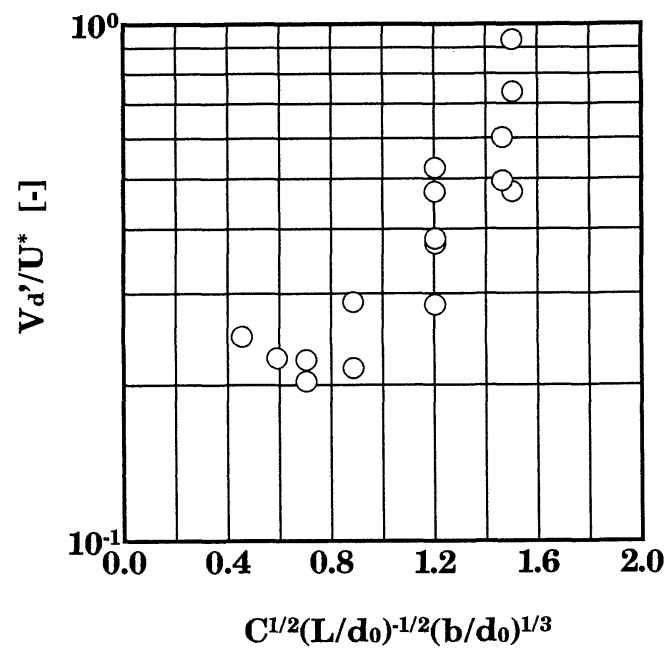

FIGURE 5 Dimensionless door jet velocity against dimensionless traveling distance modified by space factor $\mathrm{C}$ considering door width and fire source size. 


$$
\begin{aligned}
\therefore \frac{\mathbf{V}_{\mathbf{d}}}{\mathbf{U}^{*}} & =\frac{\frac{2 \cdot \mathbf{A} \cdot \mathbf{B}}{\sqrt{\mathbf{x} / \mathbf{d}_{\mathbf{o}}}} \cdot \mathbf{C}^{1 / 2} \cdot \mathbf{Q}_{\mathbf{b}}^{* / 3} \cdot\left(\frac{\mathbf{b}}{\mathbf{d}_{\mathbf{o}}}\right)^{5 / 6} \cdot\left(\frac{1}{2}\right)^{5 / 6}}{2^{1 / 3} \cdot \mathbf{Q}_{\mathbf{b}}^{* 1 / 3} \cdot \sqrt{\mathbf{g} \cdot \mathbf{b}}}=\frac{2 \cdot \mathbf{A} \cdot \mathbf{B}}{\sqrt{\mathbf{x} / \mathbf{d}_{\mathbf{o}}}} \cdot \mathbf{C}^{1 / 2} \cdot\left(\frac{\mathbf{d}_{\mathbf{o}}}{\mathbf{b}}\right)^{1 / 2} \cdot\left(\frac{\mathbf{b}}{\mathbf{d}_{\mathbf{0}}}\right)^{5 / 6} \cdot\left(\frac{1}{2}\right)^{1 / 2} \\
& =\sqrt{2} \cdot \mathbf{A} \cdot \mathbf{B} \cdot \mathbf{C}^{1 / 2} \cdot\left(\frac{\mathbf{x}}{\mathbf{d}_{\mathbf{o}}}\right)^{-1 / 2} \cdot\left(\frac{\mathbf{b}}{\mathbf{d}_{\mathbf{o}}}\right)^{1 / 3}
\end{aligned}
$$

where taking $A=5, B=0.60 .7$ based on the experimental results,

$$
\approx \mathbf{C}^{1 / 2} \cdot\left(\frac{\mathbf{x}}{\mathbf{d}_{0}}\right)^{-1 / 2} \cdot\left(\frac{\mathbf{b}}{\mathbf{d}_{0}}\right)^{1 / 3}
$$

FIGURE 5 shows the dimensionless door jet velocity against traveling distance, L, modified with the space factor $\mathrm{C}$ considering opening width. This Figure shows that the door jet is maintained as almost constant near to the door and then decreases in proportion to $\mathrm{L}^{-1 / 2}$ as we assumed.

\subsection{Inclination Angle Depend on Opening Width}

a) One Door Jet

One opening, $30 \mathrm{~cm}(\mathrm{~W}) \times 22.5 \mathrm{~cm}(\mathrm{H})$, resulted in one induced door jet which flew into the atrium space growing its width, $\sigma$, and reducing its main velocity, $V_{d}$ ', for downstream along the trajectory. The decreasing behavior of the main velocity is illustrated in FIGURE 6(a). These obtained velocities are compared with the estimated ones based on equation (2). The comparison between experimental data and the calculated one suggested that the empirical equation (2) is useful to estimate the door jet velocity which attacks a flame if we know the door jet velocity at the opening, $d_{0}$. Inclination angle, $\theta$, depends not only the door jet velocity, $V_{d}$, but also on the width of the opening. The width of one door

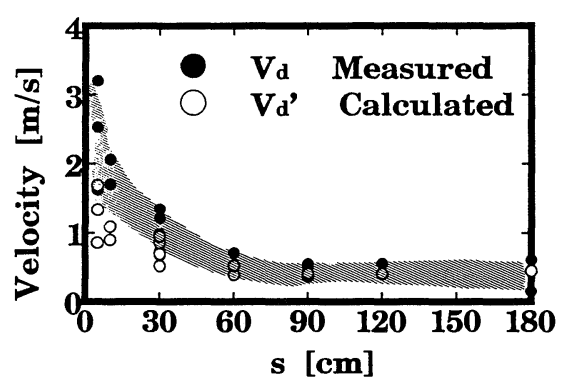

FIGURE 6 (a) Door jet velocity against traveling distance. Measure data are plotted by and estimated are plotted by $\bigcirc$.

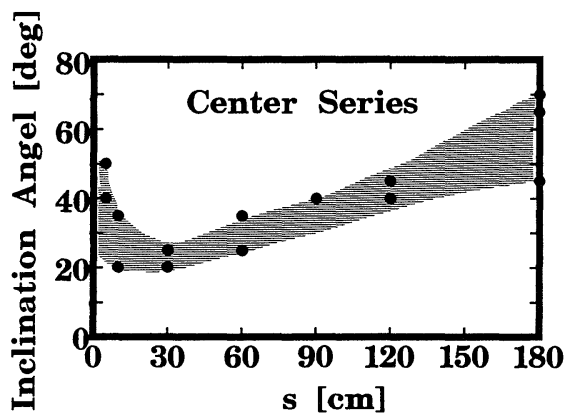

FIGURE 6(b) Inclination angle of the flame as a function of one door width. 
was changed from $5 \mathrm{~cm}$ to $180 \mathrm{~cm}$ (full open of the side wall) and also the inclination flame angle was observed and shown in FIGURE 6(b).

\section{b) Two Door Jets}

In our tests, a height of the opening was set $22.5 \mathrm{~cm}$ as the same as one door case and opening width was set half of the one opening of $15 \mathrm{~cm}$ for each opening. Both total opening area and the heat release rate are set as the same as one opening case. The door jets behavior showed roughly three phases.

As the separation is set smaller than twice one door width, merging of two jets occurred and was observed clearly. The merged flow attacked the flame similar to the one door jet, resulting in blow down for downstream direction as shown FIGURE 7(b). This situation could be assigned as the first phase, and the estimation of $V_{d}$ ' based on equation (2) is effective as shown in FIGURE 7(a). As the separation distance between two doors, $\alpha$, increased, the interaction between two jets got weaker and less merging occurred. However, the merged jets act as one jet to the flame and flame blew in the downstream direction. As the separation became wider (to about $80 \mathrm{~cm}$ in our case), the flame tended to stand up vertically. This situation was assigned as the second phase. When the separation distance of $\alpha$ became greater than about six times (about $80 \mathrm{~cm}$ in our case) the width of one opening, the induced jets penetrated into the atrium space almost parallel each other with little merging, so that merging of two jets occurred after they passed away beyond the fire source. This

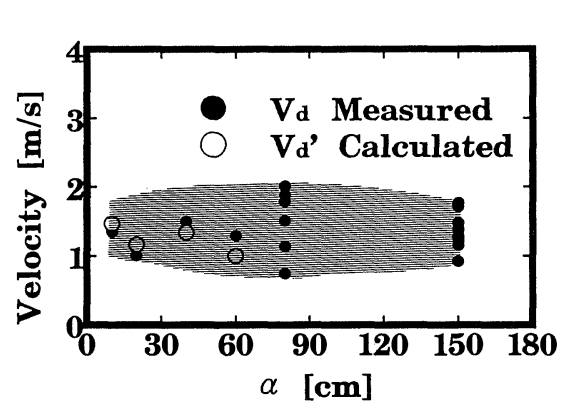

FIGURE 7 (a) Door jet velocity merged from two jets are against traveling distance. Measured data are plotted by and estimated are plotted by $\bigcirc$.

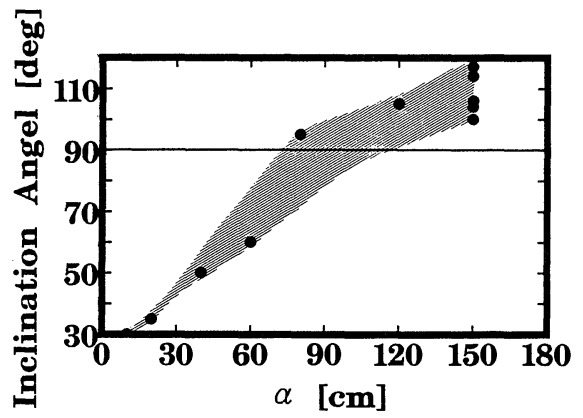

FIGURE 7 (b) Inclination angle of the flame as a function of two doors separation. Flame inclined downstream direction when door separation is set smaller than twice width of one door 
situation, the third phase, resulted in the flame leaning toward openings. In this situation, the estimation of $V_{d}$ ' based on equation (2) is not effective, because main portion of the jets passed away without active attacking on the flame.

c) Comparison of Inclination angle measured with estimated ones

Except for the third phase described in the previous section, the velocity of induced door jet(s) which attack the flame can be estimated for the one door as well as two doors case. Employing equations (1), (2), and (6), the inclination angle of the flame in the reduced atrium model with designed heat release rate of $12 \mathrm{~kW}$ was estimated versus the configuration of door width and its separation distance. FIGURE 8 shows the estimated inclination angle of the flame as a function of dimensionless door jet velocity, $V_{d}{ }^{\prime} U^{*}$. The curve in this figure was calculated based on equation (1). Comparison of experimental data for both cases of one door ( $\bigcirc$ Center-series) and two doors ( $\alpha$-series) showed the simple estimation model of flame inclination as a function of door jet. This model correlated with the atrium space and heat release rate and is useful to assess the flame inclination and fire safety.

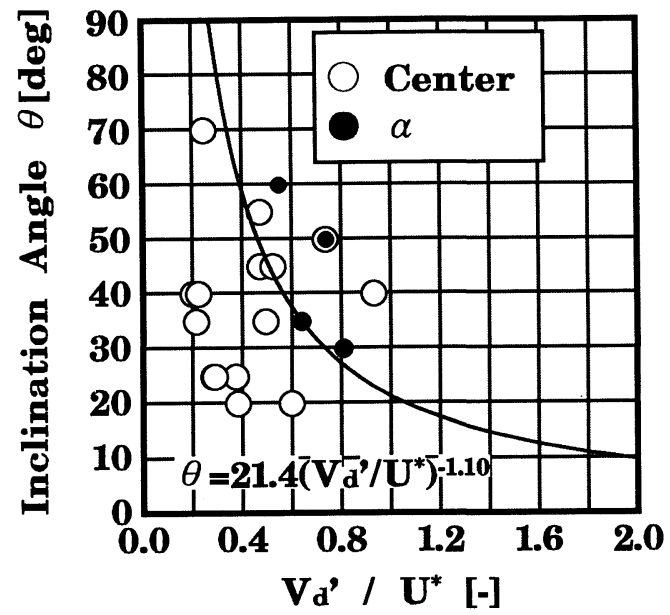

FIGURE 8 Inclination angles are plotted against the dimensionless door jet as it attacked on the flame. The superimposed curve is calculated based on equation (1). One door case which located at the center of the wall (O) and two doors case are plotted $(O$ as called $\alpha$-series tests).

\section{Conclusion}

Flame inclination behavior due to wind in open space is expressed empirically as; $\theta=$ $21.4\left(\mathrm{~V} / \mathrm{U}^{*}\right)^{-1.1}$ for the diffusion flame with floor. Inclination flame angle without floor showed similarities to the above equation, but its maximum flame inclination angle seemed to be terminated around 30 degrees and was larger than the without floor case.

The induced door jet showed normal distribution and its main velocity decreased with an increase of $1 / 2$ power of traveling distance along the trajectory. The induced velocity at the opening was estimated When two openings were set, two jets were induced and they merged together keeping their normal distributions. However, the separation distance between two openings is wider than twice the width of one opening, when interaction of two jets got weak resulting in little merging. Flow behavior of jets changed the flame inclination 
The simple model for the induced door jet velocity at the opening was established using terms for the dimensionless heat release rate, space factor of the atrium, and configuration of inlet opening(s). Considering the decreasing mode of induced jet velocity for traveling distance, $x$, the jet velocity which attacks the flame was estimated as

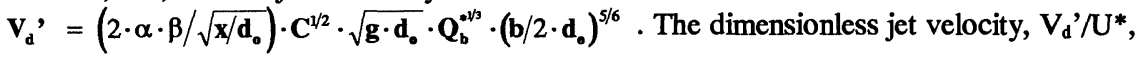
was expressed empirically as $\mathbf{V}_{d}^{\prime} / \mathbf{U}^{*}=C^{1 / 2} \cdot\left(\mathbf{x} / \mathbf{d}_{0}\right)^{-1 / 2} \cdot\left(\mathbf{b} / \mathbf{d}_{0}\right)^{1 / 3}$. The simple model was coupled with the flame inclination model, and flame inclination was estimated and compared with measurements. Comparison between estimated inclination angle and measured one verified the validity of the model presented here.

Flame inclination was shown bo be a function of location and number of opening(s). This implied that we need more basic information on flow behavior of induced door jets(s) to further assess the fire safety aspects of atrium fire.

\section{References}

1) Satoh. H., Sugawa. O., and Kurioka. H., "Plume Behavior in a Confined Tall and Narrow Space - as One of Sub-Models of Plume for an Atrium -", Proc. 4th Int. Symp. on Fire Safety Science, pp.551-562, Ottawa, (1994) and

H. Satoh, O. Sugawa, and H.Kurioka, "Modeling on Temperature and Ventilation for Fire in a Tall and Narrow Atrium (in Japanese)”, J. Archit., Plann. Envirn. Eng., AIJ, No.463, 1-10, (1994),

2) Hamada, M., "Study on Flame Inclination" Bull. Fire Prevention Society of Japan, Vol.1, No.2, pp.41-43 (1952) (in Japanese)

3) Thomas, P.H. "Some Aspect of the Growth and Spread of Fire in the Open", vol.40, No.2, Forestry, pp.140-164, (1967)

4) Escudier, M. P. "Aerodynamics of a Burning Turbulent Gas Jet in a Cross Flow", Combustion Science and Technology, vol.4, pp.293-301, (1972)

5) Brzustowski, T. A. "Hydrocarbon turbulent diffusion flame in subsource cross flow" in Reference [7]

6) Raj. P. P. K., Moussa, A. N., and Aravamudau, K. "Experiments Involving Pool and Vapor Fires from Spills of liquefied Natural Gas on Water", U.S. Coast Guard Report No. CG-D-55-77, (1979) in Reference [7]

7) Quintiere, J. G. "The Effect of Room Openings on Fire Plume Entrainment", Combustion Science and Technology, vol.26, pp.193-201, (1981) 
7. Nomenclature:

A: area of atrium floor $\left[\mathrm{m}^{2}\right]$

$\mathrm{A}_{\mathrm{d}}$ : area of inlet opening $\left[\mathrm{m}^{2}\right]$

$A_{e}$ : area of outlet/exhaust opening $\left[\mathrm{m}^{2}\right]$

$A_{T}$ : interior area of atrium $\left[\mathrm{m}^{2}\right]$

$b_{0}$ : characteristic length of fire source $[\mathrm{m}]$

$C$ : space factor for an atrium concerned, $C=\left(j / C_{D} m\right)^{2 / 3}\left(2 K^{2} / 1+K^{2}\right)^{2 / 3}\left(H^{2} / A\right)^{1 / 3}[-]$

$\mathrm{C}_{\mathrm{D}}$ : drag coefficient at inlet opening [-]

Cp: specific heat $[\mathrm{kj} / \mathrm{kg} \cdot \mathrm{K}]$

$D_{d}$ : characteristic length of fire source defined as $D_{d}=A_{d}{ }^{1 / 2}[\mathrm{~m}]$

$\mathrm{d}_{\mathrm{o}}$ : opening width [m]

g: acceleration constant due to gravity $\left[\mathrm{m} / \mathrm{sec}^{2}\right]$

$\mathrm{H}$ : height of atrium space [m]

$\boldsymbol{h}_{\mathrm{e}}$ : heat transfer coefficient $\left[\mathrm{kW} / \mathrm{m}^{2} \cdot \mathrm{K}\right]$

$\mathrm{j}$ : characteristic scale ratio, height of inlet to exhaust opening against atrium height

L: traveling distance along the trajectory [m]

$\mathbf{M}_{\mathrm{d}}$ : ascending mass flux in atrium $[\mathrm{kg} / \mathrm{sec}]$

$\mathrm{m}$ : ratio of door area to floor area, $\mathrm{m}=\mathrm{D}_{\mathrm{d}} / \mathrm{A}^{1 / 2}$

$\mathrm{m}_{\mathrm{i}}$ : distance to a trajectory of the door jet from the reference wall [m]

(defined in FIGURE 3(a))

$\mathrm{T}_{\mathrm{a}}$ : ambient temperature $[\mathrm{K}]$

$\mathrm{T}_{\mathrm{s}}$ : smoke/hot layer temperature $[\mathrm{K}]$

$\mathrm{U}^{*}$ : flame velocity based on heat release rate $[\mathrm{m} / \mathrm{s}]$, defined as $\mathrm{U}^{*}=\left(\mathrm{g} \cdot \mathrm{Q}_{\mathrm{f}} / \mathrm{b}_{\mathrm{o}} \cdot \rho \cdot \mathbf{T} \cdot \mathbf{C p}\right)^{1 / 3}$

$\mathrm{V}$ : horizontal wind velocity $[\mathrm{m} / \mathrm{s}]$

$\mathrm{V}_{\mathrm{d}}$ : induced door jet velocity as attack on a flame $[\mathrm{m} / \mathrm{s}]$

$\mathrm{Q}_{\mathrm{f}}$ : heat release rate from a model fire source $[\mathrm{kW}]$

$\mathrm{Q}_{\mathrm{fd}}{ }^{*}$ : dimensionless heat release rate, defined as $\mathrm{Q}_{\mathrm{fd}}{ }^{*}=\mathrm{Q}_{\mathrm{f}} / \mathrm{Cp} \cdot \rho_{\mathrm{a}} \cdot \mathrm{T}_{\mathrm{a}} \cdot \mathrm{g}^{1 / 2} \cdot \mathrm{D}_{\mathrm{d}}{ }^{5 / 2}$

$K^{2}=\left(\mathbf{T}_{\mathrm{a}} / \mathbf{T}_{\mathrm{s}}\right)\left(\mathbf{A}_{\mathbf{e}} / \mathbf{A}_{\mathrm{d}}\right)^{2}$

$\mathrm{x}$ : axis along the traveling distance from the reference wall where locates inlet door opening $[\mathrm{m}]$

$\mathrm{y}$ : axis from the reference wall perpendicular to $\mathrm{x}[\mathrm{m}]$

$\mathbf{Y}^{*}$ : dimensionless heat loss coefficient defined as $\mathbf{Y}^{*}=\mathbf{1}+\boldsymbol{h}_{e} \mathbf{A}_{\mathbf{T}} /\left(\mathbf{M}_{\mathrm{d}} \mathbf{C}_{\mathbf{p}}\right)$

$\alpha$ : separation distance between two openings for the $\alpha$-series experiment [m] (defined in FIGURE 3(a))

$\sigma:$ characteristic width of door jet [m]

$\theta$ : flame inclination angle [degree]

$\rho:$ density $\left[\mathrm{kg} / \mathrm{m}^{3}\right]$ 